560

ANALYSIS OF 168 PRIMARY ADULT OVARIAN GRANULOSA CELL TUMORS, ITS CLINICOPATHOLOGICAL OUTCOME, AND RISK FACTORS FOR RECURRENCE

1,2 $\mathrm{H}$ Plett* ${ }^{*}, 4 \mathrm{E}$ Ricciardi, $2,5 \mathrm{JP}$ Ramspott, ${ }^{6,7} \mathrm{~V}$ Vacaru, ${ }^{4} \mathrm{~N}$ Colombo, ${ }^{2} \mathrm{~A}$ Du Bois, ${ }^{6} \mathrm{~J}$ Sehouli, ${ }^{2} \mathrm{~A}$ Traut, ${ }^{6} \mathrm{R}$ Richter, ${ }^{2} \mathrm{P}$ Harter. ${ }^{1}$ University Hospital Leipzig, Department of Gynecology, Leipzig, Germany; ${ }^{2}$ Ev. Kliniken Essen-Mitte, Department of Gynecology and Gynecologic Oncology, Essen, Germany; ${ }^{3}$ Ospedale Sandro Pertini, Gynecologic Oncology Unit, Department of Obstetrics and Gynecology, , Roma, Italy; ${ }^{4}$ European Institute of Oncology, Italy; ${ }^{5}$ University Hospital Münster - UKM, Department of Gynecology and Obstetrics, Münster, Germany; ${ }^{6}$ Charité Campus Virchow Clinic, Department of Gynecology with Center for Oncological Surgery, Berlin, Germany; ${ }^{7}$ Vivantes Humboldt-Klinikum, Berlin, Germany

\subsection{6/ijgc-2021-ESGO.429}

Introduction/Background* Adult granulosa cell tumors (aGCT) represent less than $5 \%$ of all ovarian malignancies. The aim of this study was to analyze clinical and histopathological parameters and their impact on recurrence, progression-free(PFS) and overall survival (OS).

Methodology Patients diagnosed with primary aGCT and treated in three international referral centers were included in the study. The following variables were anonymously exported from the prospective database of each clinic for further analysis: patient's age at diagnosis, stage, chemo-, radiation, or hormonal therapy, surgery for primary site, type of restaging surgery, lymph nodes dissected, follow-up months, PFS, and OS. Descriptive statistical analysis regarding tumor and treatment characteristics was performed. Survival analyses included Kaplan-Meier functions and Cox proportional hazard ratios (HR).

Result(s)* The total study cohort included 168 patients with primary aGCT, which were treated surgically. Median age was 50 years (range 13-82). 54.2\% $(\mathrm{n}=91)$ of patients had FIGO stage IA, $26.8 \%(n=45)$ were stage IC, and $17.8 \%(n=32)$ had FIGO stage II-IV. In total $66.7 \%(n=112)$ of patients underwent surgical restaging procedure of whom $11.9 \%$ $(n=20)$ were up-staged. A median laparotomy was performed in $70.8 \% \quad(n=119)$ of patients. Adjuvant chemotherapy was administered to $11.3 \%$ of patients $(n=19)$ and one patient received endocrine therapy. After a median follow-up of 61 months, $10.7 \%(n=18)$ had recurrent disease and $4.8 \%(n=8)$ of patients died from the disease. Overall five-year PFS was $86.1 \%$ and estimated OS was 95.7\%. Survival was worse for patients with advanced stages (FIGO IA/B vs. IC, HR = 5.09; 95\% confidence interval [CI]: 1.53-16.9; FIGO IA/B vs. II-IV, $\mathrm{HR}=5.62$; 95\% CI: 1.58-19.9) and for patients who underwent adjuvant chemotherapy $(\mathrm{HR}=9.15$; 95\% CI: 3.62-23.1). Conclusion* Prognosis of patients with primary aGCT is mainly determined by FIGO-Stage. The outcome of FIGO stage IC is comparable to advanced stages and a significant number of patients were up-staged. The role of adjuvant chemotherapy remains unclear and should be investigated in future studies.

\section{OVERALL SURVIVAL RATES AND PROGRESSION FREE SURVIVAL IN IDS VS PDS IN ADVANCED OVARIAN CANCER PATIENTS IN THE UNIVERSITY HOSPITALS OF LEICESTER}

A Barakat*, A Ismail, Q Davies, S Chattopadhyay. University Hospitals of Leicester, GynaeOncology, Leicester, UK
Introduction/Background* Surgery is known to be a prognostic factor in patients with advanced ovarian cancer. However, how does the overall survival rates compare in patients undergoing Interval debulking surgery versus primary debulking surgery.

Methodology A retrospective study was done involving 102 advanced ovarian cancer patients (FIGO stage $3 \mathrm{c}$ and 4) undergoing IDS and PDS in the UHL between January 2015 to January 2020. The number of patients recruited for the study were 57 and 45 patients undergoing interval debulking surgery (IDS) and primary debulking surgery (PDS) respectively. The objective of the study is to explore and compare the survival outcomes in both groups.

Result(s)* Advanced ovarian cancer patients undergoing IDS have 1-year, 3-year and 5-year overall survival rates of $94.7 \%$, $49.2 \%$ and $36.9 \%$, while those undergoing PDS have overall survival rates of $84.4 \%, 68.9 \%$ and $61.3 \% \quad(p=0.081)$ generally.

The 1-year, 3-year and 5-year overall survival rates in FIGO stage $3 \mathrm{c}$ advanced ovarian cancer patients was significantly higher in the PDS group in contrast to IDS patients. In fact, the overall survival rates are $92.3 \%, 48.3 \%$ and $38.6 \%$ in patients who underwent IDS and 91.2\%, 77.1\% and $67.5 \%$ in patients who underwent PDS $(p=0.019)$. This was

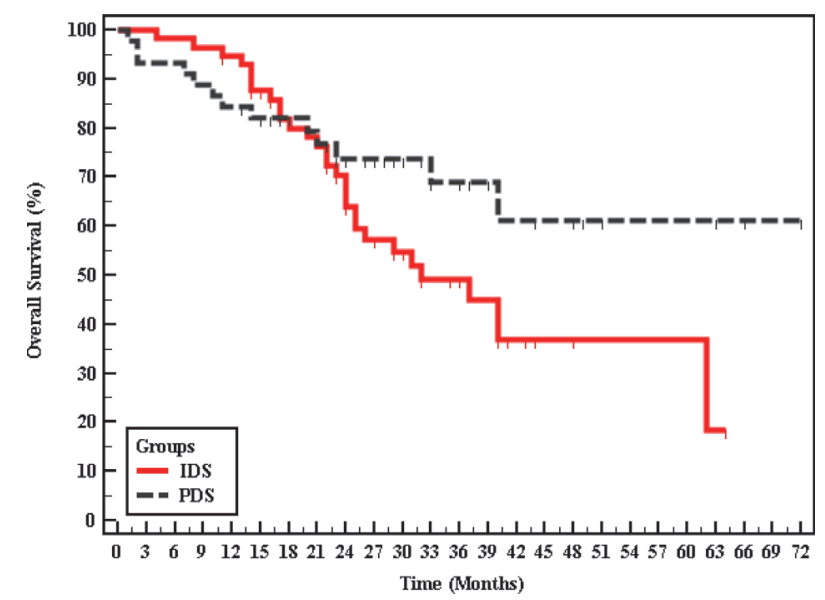

Abstract 562 Figure 1 Kaplan-Meier survival curve for overall survival with groups in total stage

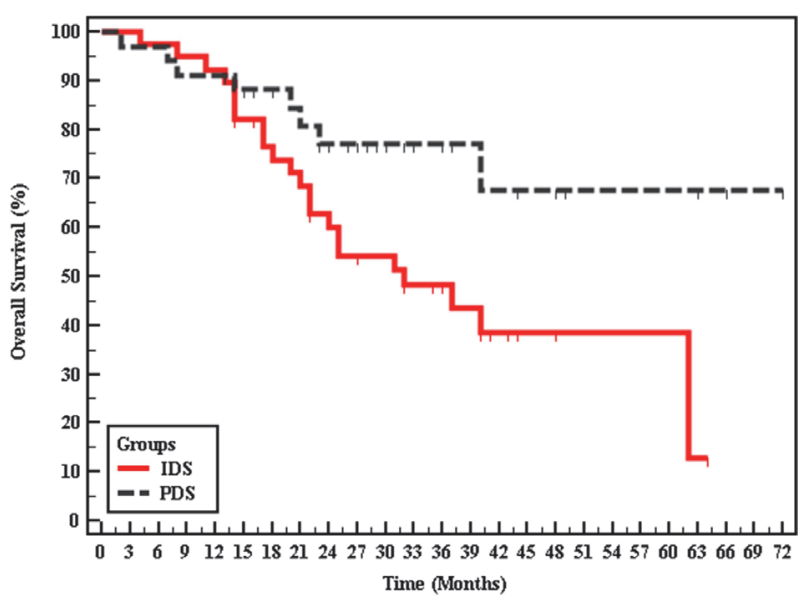

Abstract 562 Figure 2 Kaplan-Meier survival curve for overall survival with groups in stage 3 